\section{Regards sur l'économie allemande}

Bulletin économique du CIRAC

$86 \mid 2008$

Varia

\title{
Fret ferroviaire en RFA : une dynamique à optimiser
}

Isabelle Bourgeois

\section{OpenEdition}

\section{Journals}

Édition électronique

URL : http://journals.openedition.org/rea/886

DOI : $10.4000 /$ rea. 886

ISBN : 978-2-8218-0868-3

ISSN : 1965-0787

\section{Éditeur}

CIRAC

Édition imprimée

Date de publication : 1 mai 2008

Pagination : 5-18

ISSN : 1156-8992

\section{Référence électronique}

Isabelle Bourgeois, «Fret ferroviaire en RFA : une dynamique à optimiser », Regards sur l'économie allemande [En ligne], 86 | mai 2008, document 1, mis en ligne le 01 mai 2010, consulté le 30 avril 2019. URL : http://journals.openedition.org/rea/886 ; DOI : 10.4000/rea.886 


\section{Fret ferroviaire en RFA : une dynamique à optimiser}

\section{Isabelle Bourgeois}

La compétitivité de l'économie allemande, largement ouverte aux échanges, repose foncièrement sur les performances du secteur de la logistique et, en son sein, des activités de transport de fret, elles-mêmes dépendantes de la qualité des infrastructures. Or dans l'espace communautaire élargi, et du fait de l'accélération des échanges mondiaux, les flux de marchandises sont en forte croissance tout en se diversifiant. C'est la raison pour laquelle l'un des axes prioritaires de la politique du gouvernement fédéral est de créer les conditions pour pérenniser "la position de leader de l'Allemagne en tant que site logistique " dans l'UE en « accroissant l'efficience du système global du transport de fret et en favorisant une meilleure utilisation des infrastructures de transport ", ainsi que le formule le Contrat de coalition conclu le 11 novembre 2005. Cette politique, caractérisée par une approche globale de la mobilité des biens et personnes, intègre de nombreux aspects dont la modernisation et l'extension des infrastructures.

Cette question est cruciale pour l'Allemagne : depuis l'Unité, le développement des infrastructures dans les nouveaux Länder était prioritaire ; or dans le même temps, l'accélération de l'intégration du marché communautaire et l'élargissement de l'UE à l'est décuplaient le trafic sur le territoire allemand, multipliant les goulots d'étranglement à l'ouest comme à l'est; enfin, la montée en puissance des échanges avec les économies émergentes et sa contribution à l'essor du transport combiné (conteneurs) rend urgente aujourd'hui le réaménagement de l'hinterland des ports maritimes. Ces évolutions forcent une approche de la politique des transports sous l'angle de son impact sur une économie structurellement dépendante des échanges, telle que l'a présentée en mars 2008 le ministère fédéral des Transports via son projet de "Masterplan pour le transport de marchandises et la logistique ", contribution allemande à l'agenda communautaire visant à " renforcer l'efficacité, l'intégration et le caractère durable du transport de marchandises en Europe » (COM [2007] 606 final).

Dans le cadre de cette approche par nature intermodale, une attention particulière est portée au rail - un mode performant, puisqu'il transporte un sixième du fret recensé outre-Rhin, et que le quart du transport ferroviaire de marchandises de l'UE est à mettre à l'actif de l'Allemagne. Mais son énorme potentiel, en complémentarité avec les autres modes de transport dans un Etat qui est la principale plate-forme logistique de l'UE, est encore largement bridé par une série de contraintes. La principale réside dans l'absence de choix politiques clairs qui font que la privatisation de la société des chemins de fer Deutsche Bahn AG, bien que programmée de longue date, est sans cesse repoussée et que la libéralisation du secteur du fret ferroviaire est ainsi restée à mi-chemin.

L'Allemagne est la première 'plate-forme logistique' de l'Europe, avec un cinquième du CA total du secteur de la logistique réalisé au sein de l'UE (730 milliards $€$ en 2004 dans l'UE 15 plus la Suisse et la Norvège ; Klaus, Kille, 2006). Cette branche est encore imparfaitement prise en considération par les statistiques officielles puisqu'une majorité de ses activités se situe à l'interface entre les services et l'industrie, ce qui soulève un problème de délimitation : Destatis ne prend ainsi en compte que les activités de logistique - surtout de transport -

Allemagne, principale plate-forme logistique' de I'UE 
Logistique en RFA : au même rang que la construction mécanique

Un réseau d'infrastructures propice à l'intermodalité externes à l'industrie, or celle-ci en effectue plus de la moitié en interne (transport inter-sites). "Le transport de marchandises et la logistique constituent une base importante de notre prospérité, mais dont la portée est rarement estimée à sa juste valeur », explique le ministère fédéral des Transports dans son projet de Masterplan Güterverkehr und Logistik - Entwurf, 2008.

La logistique est en effet l'activité sur laquelle repose l'ensemble de la structure économique du pays. Selon les estimations du groupe de travail « Technologies des services de Logistique » (ATL) de l'Institut Fraunhofer Integrierte Schaltungen (IIS, Erlangen), qui a collecté les données éparses et partielles à diverses sources, cette 'branche' se situe en troisième position en termes de chiffre d'affaires dans la structure économique de l'Allemagne, au même rang que la construction mécanique (170 milliards $€$ en 2004) et après l'industrie automobile et le secteur de la santé (respectivement 285 milliards $€$ et 250 milliards $€$; Klaus, Kille, 2006). Elle génère 2,5 millions d'emplois directs, auxquels il faut ajouter un demi million d'emplois indirects. Les données avancées par le projet de «Masterplan », basées sur les estimations de cette étude, confirment la position de leader européen de l'Allemagne : elle réalise quelque $21 \%$ (189 milliards $€$ ) du CA de la logistique, évalué à 836 milliards $€$ pour l'UE 27 (plus la Norvège et la Suisse) en 2006.

Près de la moitié de ce CA est générée par les seules activités de transport (44\% en 2004 ; Klaus, Kille, 2006). Il est vrai que la position géographique de l'Allemagne au centre de la «banane bleue » où se concentre l'activité dans I'UE est un facteur favorable. Or non seulement, le site Allemagne est à l'intersection des principaux axes est-ouest et nord-sud au sein de l'espace européen, mais son réseau d'infrastructures présente un maillage très dense et favorable à l'intermodalité, car conçu dès l'origine sur la mise en réseau de modes complémentaires. Avec plus de $41500 \mathrm{~km}$ de voies selon Destatis (contre 29200 en France ; Sénat, 2008), le réseau ferré allemand est le plus dense de l'UE, avec $122,7 \mathrm{~m} / \mathrm{km}^{2}$, dont près de la moitié de lignes à deux voies ou plus (IFMO, 2007). S'y ajoutent plus de $230000 \mathrm{~km}$ de routes d'importance régionale et nationale (dont $12000 \mathrm{~km}$ d'autoroutes), et $7300 \mathrm{~km}$ de voies de navigation intérieures débouchant sur le port fluvial de Duisburg (premier mondial) comme sur les ports maritimes internationaux de Rotterdam (Pays-Bas) ou de Hambourg et Bremerhaven (les deux plus grands ports maritimes de conteneurs d'Allemagne), sans oublier les plate-formes aéroportuaires comme Francfort/Main, Munich ou Düsseldorf. Et il est vrai également que les secteurs à forte croissance, notamment les activités industrielles de moyenne et haute technologie, se concentrent près des points nodaux intermodaux de ce réseau d'infrastructures (Bourgeois, 2007).

Transport de marchandises en Allemagne

\begin{tabular}{|l|cc|cc|cc|}
\hline Mode & $\begin{array}{c}\mathbf{2 0 0 7} \\
\text { Tonnes } \\
\text { (en mio) }\end{array}$ & $\begin{array}{c}\text { Tkm } \\
\text { (en } \mathrm{mrd} \text { ) }\end{array}$ & $\begin{array}{c}\mathbf{2 0 0 3} \\
\text { Tonnes } \\
\text { (en mio) }\end{array}$ & $\begin{array}{c}\text { Tkm } \\
\text { (en } \mathrm{mrd} \text { ) }\end{array}$ & $\begin{array}{c}\mathbf{2 0 0 0} \\
\text { Tonnes } \\
\text { (en mio) }\end{array}$ & $\begin{array}{c}\text { Tkm } \\
\text { (en } \text { mrd })\end{array}$ \\
Routier & 3429,6 & 466,2 & 2909,0 & 358,0 & 3005,1 & 280,7 \\
Fluvial & 358,2 & 114,0 & 297,0 & 78,5 & 294,2 & 76,0 \\
Maritime & 247,4 & 65,0 & 218,0 & 58,6 & 242,2 & 66,5 \\
Oléoducs & 311,1 & - & 253,0 & - & 238,3 & - \\
Aérien & 91,2 & 16,1 & 92,0 & 15,5 & 89,4 & 15,0 \\
Total & 3,4 & - & 2,3 & - & 2,2 & - \\
\hline
\end{tabular}

Source des données : Destatis (www.destatis.de).

Parmi les différents modes, comme dans l'ensemble du marché communautaire, la route se taille la part du lion, avec quelque $80 \%$ du tonnage et environ $70 \%$ du nombre de tonnes-kilomètres (tkm) en Allemagne. Les parts du rail se situent respectivement à $16 \%$ et $18 \%$, celles du fluvial étant de $12 \%$. D'ici 2025 , cette répartition entre les trois principaux modes de transport terrestres ne devrait pas connaître de changement significatif, ainsi qu'il ressort d'une 
étude prévisionnelle (horizon 2025) commanditée par le ministère fédéral des Transports aux cabinets Intraplan Consult et BVU Beratergruppe Verkehr + Umwelt, et publiée en novembre 2007 (ITP/BVU, 2007). Et ce, malgré la très forte dynamique que connaît depuis le début du millénaire le transport de marchandises du fait de l'accélération de l'intégration européenne, de l'élargissement à l'est de l'UE, ainsi que de la reconfiguration du partage du travail dans le fil de la globalisation des activités.

Evolution du fret en tkm et évolution des distances parcourues 2004-2025

\begin{tabular}{|c|c|c|c|c|c|c|c|c|}
\hline & \multicolumn{5}{|c|}{ Performances (tkm) } & \multicolumn{3}{|c|}{ Distances } \\
\hline & \multicolumn{2}{|c|}{2004} & \multicolumn{2}{|c|}{2025} & \multirow{2}{*}{$\begin{array}{c}\text { 2025/04 } \\
\text { évol. }\end{array}$} & \multirow{2}{*}{$\begin{array}{c}2004 \\
k m\end{array}$} & \multirow{2}{*}{$\begin{array}{c}2025 \\
k m\end{array}$} & \multirow{2}{*}{$\begin{array}{c}\text { 2025/04 } \\
\text { évol. }\end{array}$} \\
\hline & $m r d t k m$ & part & mrd tkm & part & & & & \\
\hline $\begin{array}{l}\text { Ferroviaire } \\
\text { dont combiné }\end{array}$ & $\begin{array}{l}91,9 \\
24,4\end{array}$ & $\begin{array}{l}17,6 \% \\
26,6 \%\end{array}$ & $\begin{array}{r}151,9 \\
55,9\end{array}$ & $\begin{array}{l}16,7 \% \\
36,8 \%\end{array}$ & $\begin{array}{r}65 \% \\
129 \%\end{array}$ & $\begin{array}{l}285 \\
466\end{array}$ & $\begin{array}{l}353 \\
493\end{array}$ & $\begin{array}{r}24 \% \\
6 \%\end{array}$ \\
\hline Routier longue distance & 366,5 & $70,2 \%$ & 675,6 & $74,4 \%$ & $84 \%$ & 253 & 300 & $19 \%$ \\
\hline Fluvial & 63,7 & $12,2 \%$ & 80,2 & $8,8 \%$ & $26 \%$ & 270 & 284 & $5 \%$ \\
\hline Sous-total hors fret routier courte distance & $\begin{array}{r}522,1 \\
57 ?\end{array}$ & $100,0 \%$ & 907,7 & $\begin{array}{r}100,0 \% \\
169 \%\end{array}$ & $\begin{array}{r}74 \% \\
168 \%\end{array}$ & $\begin{array}{l}260 \\
293\end{array}$ & $\begin{array}{l}306 \\
339\end{array}$ & $\begin{array}{l}18 \% \\
16 \%\end{array}$ \\
\hline Routier courte distance & 25,9 & $4,7 \%$ & 28,8 & $3,1 \%$ & $11 \%$ & 16 & 17 & $8 \%$ \\
\hline Total route & 392,5 & $71,6 \%$ & 704,3 & $75,2 \%$ & $79 \%$ & 128 & 180 & $41 \%$ \\
\hline Total & 548,1 & - & 936,5 & - & $71 \%$ & 151 & 203 & $34 \%$ \\
\hline
\end{tabular}

Source des données : ITP/BVU, Prognose der deutschlandweiten Verkehrsverflechtungen 2025.

Sous l'effet également de la libéralisation du cabotage au sein de l'UE, de l'ouverture des frontières au fret ferroviaire en janvier 2007, ainsi que sous l'essor du commerce maritime avec les économies émergentes qui dope les flux de conteneurs (faisant de Duisburg le second port de conteneurs allemand après Hambourg), les performances globales du fret (en tkm) devraient ainsi s'accroître de $71 \%$ d'ici 2025 . Cette hausse drastique du transport de marchandises, caractérisant pareillement le rail et la route, concerne et concernera moins le volume du fret (tonnage) que ses performances mesurées en nombre de tonnes-kilomètres, étant donné que les biens sont et seront transportés sur des distances de plus en plus longues. Elles devraient passer de $260 \mathrm{~km}$ en moyenne en 2004 à $306 \mathrm{~km}$ en 2025 pour les longues distances, de $151 \mathrm{~km}$ à $203 \mathrm{~km}$ pour le trafic local et régional (ITP/BVU, 2007).

Evolution de la part des types de transport dans le total du fret (2004-2025)

\begin{tabular}{|l|cc|cc|}
\hline & \multicolumn{2}{|c|}{ Volume (tonnes) } & \multicolumn{2}{c|}{ Performances (tkm) } \\
& $\mathbf{2 0 0 4}$ & $\mathbf{2 0 2 5}$ & $\mathbf{2 0 0 4}$ & $\mathbf{2 0 2 5}$ \\
Trafic intérieur & $80,0 \%$ & $71,5 \%$ & $52,6 \%$ & $43,4 \%$ \\
Sorties & $7,8 \%$ & $10,6 \%$ & $15,0 \%$ & $16,5 \%$ \\
Entrées & $8,9 \%$ & $11,8 \%$ & $17,4 \%$ & $18,8 \%$ \\
Transit & $3,3 \%$ & $6,2 \%$ & $15,0 \%$ & $21,3 \%$ \\
\hline
\end{tabular}

Source des données : ITP/BVU, Prognose der deutschlandweiten Verkehrsverflechtungen 2025.

Types de transport et modes en 2007 (et évol. 2007/06 en \%)

\begin{tabular}{|c|c|c|c|c|c|c|}
\hline & & Total & Trafic intérieur & Sorties & Entrées & Transit \\
\hline \multirow{2}{*}{ Ferroviaire } & mio de tonnes & $361,1(+4,3 \%)$ & $226,3(+3,9 \%)$ & $56,4(+3,3 \%)$ & $59,1(+5,3 \%)$ & $19,3(+10,5 \%)$ \\
\hline & mrd de $t k m$ & $109,5(+3,8 \%)$ & $48,4(+3,3 \%)$ & $25,0(+4,0 \%)$ & $23,0(+3,7 \%)$ & $13,1(+5,4 \%)$ \\
\hline \multirow{2}{*}{ Fluvial } & mio de tonnes & $249,3(+2,4 \%)$ & $59,0(+3,3 \%)$ & $59,5(+6,8 \%)$ & $107,8(+0,8 \%)$ & $22,9(-3,2 \%)$ \\
\hline & mrd de $t k m$ & $64,1(+1,1 \%)$ & $11,6(+0,2 \%)$ & $14,0(+1,1 \%)$ & $23,5(+0,8 \%)$ & $15,0(+2,2 \%)$ \\
\hline Maritime & mio de tonnes & $315,1(+4,1 \%)$ & $8,0(+9,8 \%)$ & $118,9(+1,7 \%)$ & $188,2(+5,4 \%)$ & - \\
\hline \multirow{2}{*}{ Route } & mio de tonnes & $3315,9(+1,8 \%)$ & $2806,4(+1,4 \%)$ & $208,7(+4,0 \%)$ & $198,5(+3,4 \%)$ & $102,2(+5,7 \%)$ \\
\hline & mrd de $t k m$ & $448,6(+3,4 \%)$ & $262,6(+2,5 \%)$ & $54,9(+4,3 \%)$ & $60,9(+3,6 \%)$ & $70,2(+5,8 \%)$ \\
\hline \multirow{2}{*}{ Oléoducs * } & mio de tonnes & $95,0(+0,7 \%)$ & $22,0(+0,1 \%)$ & - & $72,9(+0,9 \%)$ & - \\
\hline & mrd de tkm & $16,1(+0,5 \%)$ & $4,8(+0,4 \%)$ & - & $11,4(+0,6 \%)$ & - \\
\hline
\end{tabular}

Sources des données : Volume (tonnes) du fret ferroviaire, fluvial et maritime : Destatis ; Performances de tous les modes (tkm) et volume du transport par oléoducs (estimations) : BVU/DLR/ISL, Gleitende Mittelfristprognose für den Güter- und Personenverkehr - Mittelfristprognose Winter 2006/2007. 
Hausse du transit et du trafic transfrontières

Prédominance du transport de biens intermédiaires, véhicules et machines
L'évolution du contexte européen et mondial se reflète dans le fait que, depuis plusieurs années, le transport intérieur de marchandises, qui représente un peu plus de la moitié du total, croît plus lentement que le transport transfrontières (entrées, sorties et transit). Ce dernier a crû de $3 \%$ par an entre 1998 et 2003, avant d'enregistrer une hausse de $9,4 \%$ l'année où l'UE a accueilli 10 nouveaux Etats membres ; en 2005 et 2006, le trafic a augmenté de 2,5\% puis de $7,7 \%$. Le seul transit a augmenté de $50 \%$ entre 1998 et 2005, et il devrait encore croître de $21 \%$ d'ici 2010 (BVU/DLR/ISL, 2007). D'ici 2025, la part du trafic transfrontières devrait ainsi passer à $57 \%$ du total en tkm (ITP/BVU 2007).

Plus que la conjoncture, la mutation des activités dans la globalisation influe sur l'évolution du type de biens transportés et donc, indirectement, sur la répartition du fret selon les divers modes. La tendance à une progression plus faible du fret en volume (en tonnes) s'explique ainsi essentiellement par le net recul des marchandises pondéreuses tels que les matériaux de construction minéraux (en baisse de $38 \%$ entre 2003 et 2005 ; Progtrans, 2007) au profit des aliments/fourrages et des produits chimiques (en hausse de $10 \%$ ), et plus encore des biens intermédiaires, véhicules et machines (+20\%).

Structure des biens transportés selon les modes en 2007

\begin{tabular}{|c|c|c|c|c|c|c|c|c|}
\hline & \multirow{2}{*}{\multicolumn{2}{|c|}{ Ferroviaire }} & \multirow{2}{*}{\multicolumn{2}{|c|}{ Fluvial }} & \multicolumn{4}{|c|}{ Routier } \\
\hline & & & & & \multicolumn{2}{|c|}{ Trafic local intérieur... } & \multicolumn{2}{|c|}{... régional/international } \\
\hline & $m r d t k m$ & $07 / 06$ & $m r d t k m$ & $07 / 06$ & $m r d t k m$ & $07 / 06$ & mrd tkm & $07 / 06$ \\
\hline Produits agricoles & 4,8 & $+3,4 \%$ & 5,4 & $+3,2 \%$ & 1,4 & $+0,6 \%$ & 37,4 & $+3,9 \%$ \\
\hline Aliments/fourrage & 1,5 & $+2,9 \%$ & 5,9 & $+2,8 \%$ & 2,2 & $+0,6 \%$ & 75,0 & $+3,7 \%$ \\
\hline Combustibles minéraux solides & 7,3 & $-3,3 \%$ & 8,2 & $-4,2 \%$ & 0,1 & $-4,8 \%$ & 1,7 & $-5,2 \%$ \\
\hline Produits pétroliers & 10,5 & $+1,2 \%$ & 10,6 & $+0,8 \%$ & 1,3 & $+0,2 \%$ & 10,5 & $+1,8 \%$ \\
\hline Minéraux, déchets métal. & 8,2 & $+0,3 \%$ & 6,1 & $-0,2 \%$ & 0,3 & $-0,4 \%$ & 6,2 & $+6,9 \%$ \\
\hline Fer, métaux non ferreux & 13,4 & $+1,6 \%$ & 3,8 & $+1,5 \%$ & 0,5 & $+0,1 \%$ & 24,6 & $+0,2 \%$ \\
\hline Mat. de construction minéraux & 8,3 & $+1,5 \%$ & 10,5 & $+0,6 \%$ & 15,6 & $+0,5 \%$ & 47,5 & $+2,0 \%$ \\
\hline Engrais & 2,2 & $+0,1 \%$ & 2,5 & $-0,7 \%$ & 0,2 & $-2,2 \%$ & 2,2 & $+4,4 \%$ \\
\hline Produits chimiques & 9,9 & $+4,4 \%$ & 5,2 & $+3,8 \%$ & 2,1 & $+1,6 \%$ & 37,1 & $+1,7 \%$ \\
\hline Autres biens finis et semi-finis & 43,4 & $+7,9 \%$ & 5,9 & $+6,1 \%$ & 3,5 & $+4,7 \%$ & 179,3 & $+4,7 \%$ \\
\hline Total & 109,5 & $+3,8 \%$ & 64,1 & $+1,1 \%$ & 27,1 & $+1,1 \%$ & 421,5 & $+3,5 \%$ \\
\hline
\end{tabular}

Source des données : BVU/DLR/ISL, Gleitende Mittelfristprognose für den Güter- und Personenverkehr-Mittelfristprognose Winter $2006 / 2007$ (estimations).

Dynamique du transport unitisé

Dans I'UE, une UTI sur trois est chargée/déchargée outre-Rhin
Ces biens, sur lesquels reposent les performances de l'industrie allemande (et européenne), tirent de leur côté la dynamique du transport unitisé, c'est-à-dire du fret en conteneurs et autres unités mobiles (unités de transport intermodales : UTI), en adéquation avec les flux tendus, le supply chain management et, par-delà, avec la tendance à la globalisation des process. La montée en puissance des UTI s'explique aussi, dans le même temps, par l'essor des échanges maritimes dans le partage mondial du travail. Ces évolutions amènent les divers acteurs de la chaîne, des industriels aux transporteurs en passant par les affréteurs, à considérer dès l'amont les modes de transport sous l'angle de leur complémentarité, ce qui force le développement du transport combiné, seul à même d'assurer la fluidité requise. De ce fait, les acteurs publics sont amenés à reconsidérer la politique des transports dans une perspective elle aussi plus globale, où les infrastructures sont le facteur déterminant pour l'activité industrielle domestique et où elles sont dans le même temps une condition sine qua non pour la compétitivité du site Allemagne dans un espace européen où les échanges sont de plus en plus fluides.

En 2007, le transport unitisé a connu outre-Rhin une forte progression sur le rail et la mer. Selon Destatis, le flux maritime d'UTI a atteint 15,24 millions d'EVP (équivalent vingt pieds, taille standard d'un conteneur), soit $+10,4 \%$ en un an. Actuellement, $85 \%$ environ du total des UTI transportées en Allemagne par route ou rail le sont dans l'hinterland des ports maritimes (BAG, 2007). D'ici 2020, les seuls ports de Bremerhaven et de Hambourg devraient, selon la « résolution » de la fédération allemande des $\mathrm{CCl}$ sur l'avenir de la politique des transports, connaître une croissance du trafic conteneur de respectivement 
$239 \%$ et $291 \%$ (DIHK, 2007). Dans le ferroviaire, la hausse de +15,9\% enregistrée en 2007, pour un total certes inférieur au flux maritime (5,6 millions d'EVP), n'en est pas moins révélatrice d'un fort potentiel. Car $44 \%$ des UTI proviennent des ports de Hambourg, Bremerhaven, Cuxhaven, Emden, Wilhelmshaven, Lübeck et Rostock ; 37 \% sont acheminées vers ceux-ci (Wirtschaft und Statistik, 6/2007).

Sur l'axe terrestre nord-sud au sein de l'UE, « le chemin de fer se taille une part élevée dans l'unitarisation ", explique Eurostat (Statistiques en bref, 20/2008). D'ici 2025, dans le seul mode rail, le transport combiné devrait doubler en tonnage comme en tkm, prévoit l'étude ITP/BVU, pour représenter le quart du volume et le tiers des performances du fret ferroviaire. Aujourd'hui, déjà, "dans le transport ferroviaire et routier du fret unitisé, l'Allemagne réalise des performances au moins deux fois plus élevées que celles de n'importe quel autre Etat membre " (Statistiques en bref, 20/2008). Avec une performance en fret unitisé de 30 millions de tkm par route et presque autant par rail en 2005 (26 millions de tkm), l'Allemagne se place en effet de loin en première position en Europe, suivie par le Royaume-Uni au second rang pour la route (12 millions de tkm) et par l'Italie pour le rail ( 9 millions de tkm) ; la France ne vient qu'en $6^{\mathrm{e}}$ (route : 7 millions de $\mathrm{tkm}$ ) et $3^{\mathrm{e}}$ position (rail : 9 millions de $\mathrm{tkm}$ ). Si on considère les trois modes rail, route et mer, "environ une UTI sur trois dans l'UE a été chargée ou déchargée » en Allemagne en 2005, souligne Eurostat. Entre la route, le rail et la mer, la complémentarité est particulièrement étroite, les modes terrestres se partageant l'acheminement sur les courtes (route) et longues (rail) distances.

\section{Renaissance du rail en Allemagne}

Depuis le début du millénaire, l'Allemagne connaît une véritable renaissance du rail, mode dopé par l'ouverture comme l'extension du marché communautaire. Alors que, dans la répartition du fret par voies modales (modal mix), la part du ferroviaire en tkm était tombée en 2002 à moins des $16 \%$ qu'il enregistrait au début des années 1990, elle s'élève continûment depuis, franchissant le seuil des $16 \%$ en 2003, montant à 16,4\% en 2005 pour dépasser $17 \%$ dès 2006 . Aujourd'hui, le quart du fret ferroviaire de l'UE est à mettre à l'actif de l'Allemagne : 107 milliards de tkm sur les 395 milliards de tkm recensés en 2006 . L'Allemagne se place ainsi au premier rang devant la Pologne (44,3 milliards de tkm) et la France (40,9 milliards de tkm ; Wirtschaft und Statistik, 6/2007).

Les performances du rail allemand ont enregistré une croissance record en $2006(+12,1 \%)$, bénéficiant d'un environnement conjoncturel porteur et d'une météo qui avait forcé le transfert sur le rail d'une partie du fret fluvial. Elles ont continué de progresser en 2007 (+7,1\%) pour atteindre 114,6 milliards de tkm (361,1 millions de tonnes de marchandises). En 1990, rappelle Destatis, ce niveau n'était encore que de 103 milliards de tkm. Quant à la distance moyenne parcourue sur le territoire allemand, elle a considérablement augmenté elle aussi, passant de 219 km en 1996 à 309 km en 2006. Ouverture intracommunautaire des frontières et élargissement de l'UE aidant, c'est le trafic ferroviaire transfrontières qui a connu la plus forte hausse, passant en l'espace de dix ans d'un peu plus de $26 \%$ à $37 \%$ : le seul transit (17,5 millions de tonnes en 2006) a augmenté de 95,9\%, suivi par les sorties (54,6 millions de tonnes, $+61,6 \%$ ) et, dans une moindre proportion, les entrées (56,1 millions de tonnes, $+36,3 \%$ ) de marchandises. Si elle reste importante en volume, la part du trafic intérieur tombait de -7,5\% pour s'établir à 217,9 millions de tonnes en 2007 (107 milliards de $\mathrm{tkm})$.

Au cours de la décennie écoulée, la structure des marchandises transportées s'est modifiée, sur le rail comme ailleurs, les biens pondéreux reculant au profit des biens de plus forte valeur. Ainsi par exemple les combustibles minéraux solides, qui représentaient encore $22 \%$ du total du volume du fret ferroviaire en 1996, sont tombés à $15 \%$ (à 51,3 millions de tonnes) ; en tkm, leur part s'est
Importance stratégique de l'axe nord-sud en Europe

Le quart du fret ferroviaire de l'UE s'effectue en Allemagne

Une croissance tirée par le trafic transfrontières

Biens intermédiaires : un gros tiers du fret 
Report partiel du fret routier vers le rail

La relocalisation des activités dans l'UE accroît la demande

réduite de $10 \%$ à $7 \%$ (7,7 milliards de tkm). A l'inverse, les biens intermédiaires et, en leur sein, les transactions spéciales, ont vu leur part passer de $15 \%$ à $24 \%$ en volume (à 81,2 millions de tonnes en 2006 ) et de $31 \%$ à $37 \%$ en performance (39,1 milliards de tkm ; Wirtschaft und Statistik, 6/2007).

Les raisons de ce regain du rail en volume comme en valeur sont de plusieurs ordres, à la fois économiques et politiques ou réglementaires, nationaux et européens. L'élargissement à l'est s'est traduit par une extension du territoire comme des activités dans l'UE ; certes, l'interconnexion des grands axes est- et ouest-européens est loin d'être achevée, et la fluidité du trafic achoppe encore sur un certain nombre de points comme l'écartement des rails ou la reconnaissance de la formation des conducteurs. Mais ces barrières sont pour l'instant compensées par une certaine complémentarité intermodale, et la dynamique est lancée. Elle est entretenue par ailleurs par un engorgement croissant des capacités routières, auquel s'ajoutent la flambée des prix des carburants ou, dans le cas de l'Allemagne, l'introduction d'un péage autoroutier pour les poids lourds en 2005, favorisant dans certains cas la substitution du rail à la route.

L'accroissement des performances du rail (en tkm) s'est traduite de son côté par une amélioration du service, le ferroviaire gagnant en régularité et prévisibilité, ce qui répond à la demande d'une industrie produisant en flux tendus et entretient aussi une tendance à la substitution intermodale pour des biens à plus forte valeur comme les machines ou pièces. La relocalisation des activités au sein de l'espace européen abonde dans le même sens, exigeant un réseau de liaisons longues distances et des dessertes fiables. Enfin, la levée progressive des barrières et l'ouverture à la concurrence des activités de fret dans l'UE, ainsi qu'en Allemagne, entretiennent elles aussi une dynamique favorable, d'autant que la position géographique de l'Allemagne au centre de la zone où se concentrent les activités dans l'UE en fait tout naturellement la plate-forme logistique centrale.

\section{Grand Axe ferroviaire (fret) Scandinavie-Rhin-Rhône-Méditerranée}

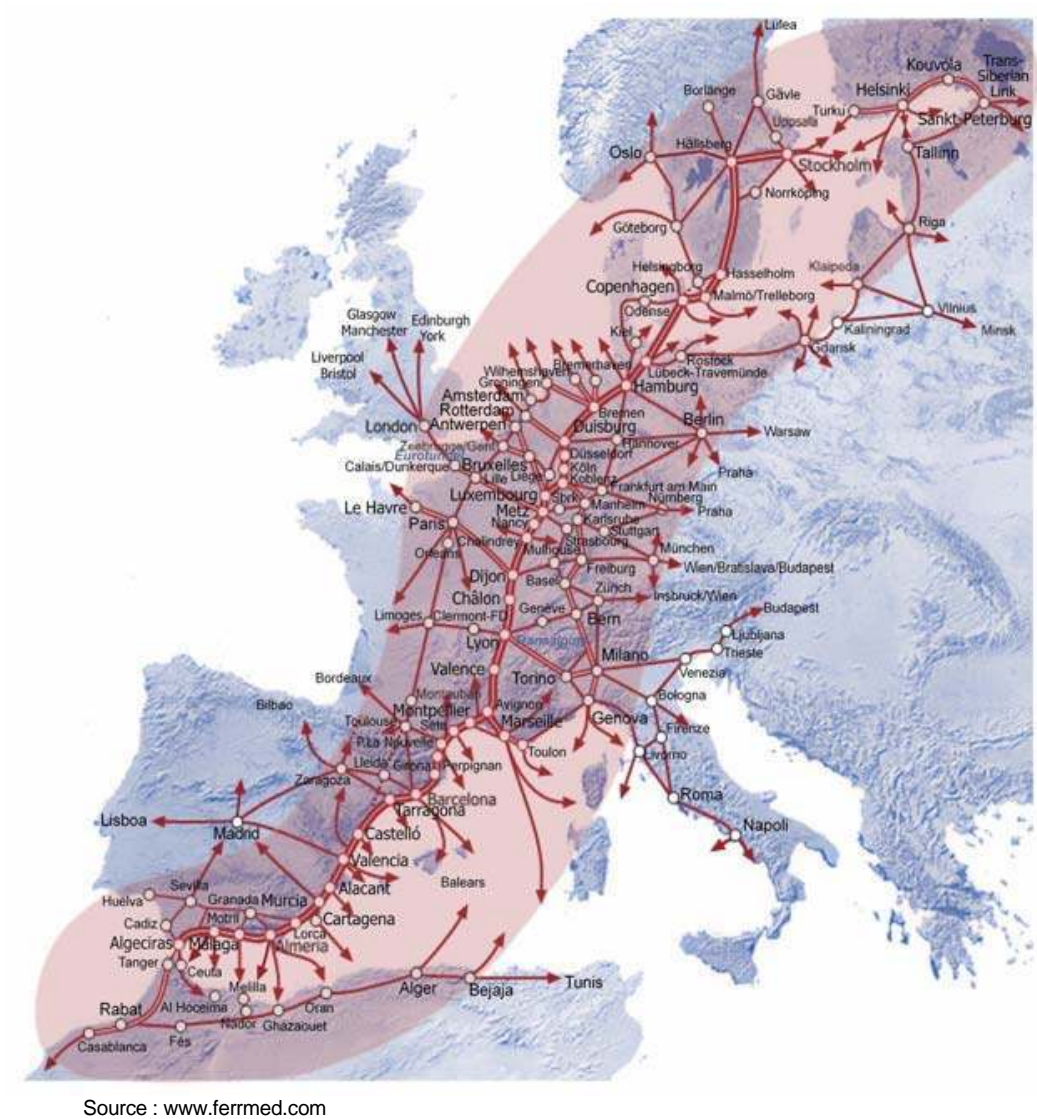

Cet axe est le plus important corridor de transport intermodal de fret en Europe. II est en partie virtuel encore, puisqu'un certain nombre de ses tronçons ne sont qu'à l'état de projet dans le cadre de la création d'un réseau transeuropéen de transport (RTE-T) à l'horizon 2020 grâce à la construction de chaînons manquants et la suppression de goulots d'étranglement dans les infrastructures existantes. Ainsi, l'axe triangulaire intermodal nordique (Finlande, Suède jusqu'à la frontière russe) ne devrait être achevé qu'après 2010. II en va de même des axes ferroviaires du détroit de Fehmarn (Copenhague-Hambourg), "Rail Baltica " (Kaunas-Riga-Tallinn-Helsinki) ou transpyrénéen Sines/Algeciras-Madrid-Paris.

En Allemagne, ce corridor Scandinavie-Méditerrannée (il résulte du maillage de divers tronçons du RTE-T) croise les liaisons existantes au sein de la "banane bleue ", cette chaîne de pôles de concentration d'activités s'étendant de la Grande Bretagne via le Benelux et l'axe du Rhin jusqu'au Nord de I'Italie. L'Allemagne (avec les Pays-Bas, où la ligne ferroviaire de Betuwe relie aujourd'hui le Rhin à Rotterdam) est également la principale zone d'interconnexion des axes multimodaux présents et futurs entre l'est et l'ouest de l'Europe. Par exemple: Rhin/Meuse-Main-Danube (fluvial) ou Athènes-Budapest-Prague-Nuremberg-Dresde (rail), eux-mêmes reliés à des transversales, par exemple Berlin-Milan-Naples via le Brenner.

L'axe ci-contre, crucial aussi pour le commerce maritime, constitue de ce fait une zone de localisation idéale pour les plate-formes logistiques intermodales. 


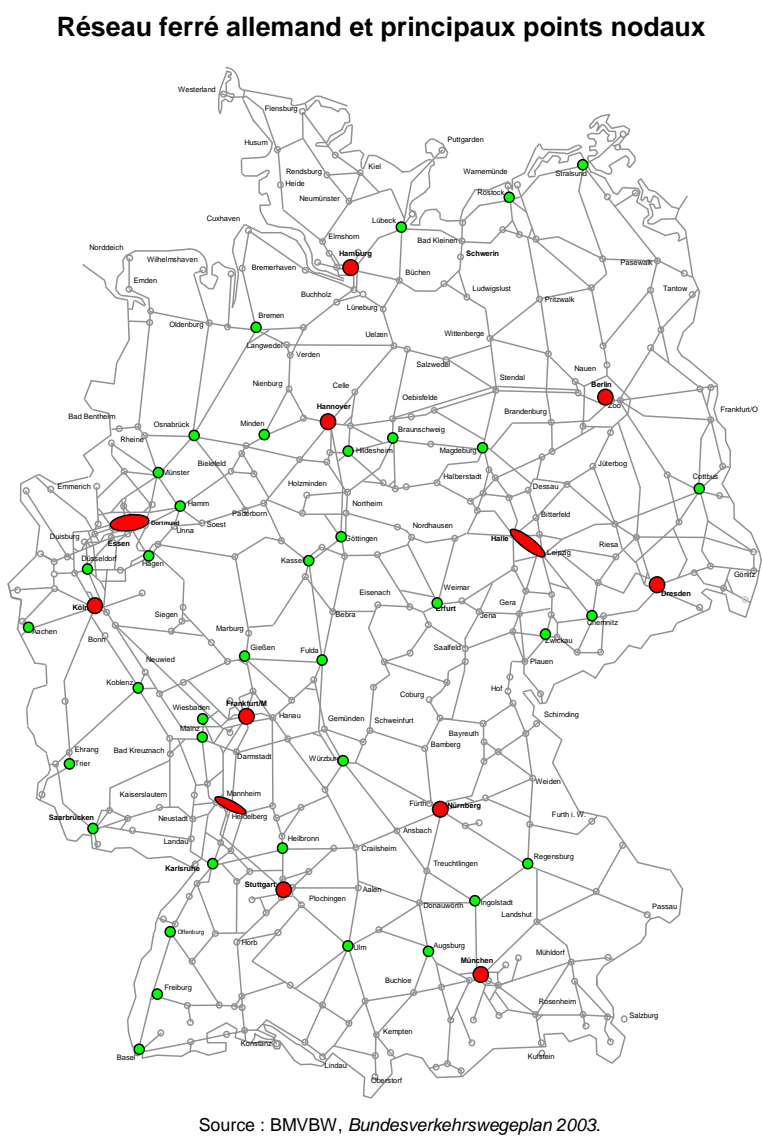

La forte croissance du fret ferroviaire enregistrée en 2006/05 (+12\% en tkm) résulte moins de la dynamique de la société publique Deutsche Bahn AG que de celle de ses concurrents ; la première n'a vu progresser ses activités que de $+9,5 \%$, contre $28 \%$ pour les derniers, selon un rapport de l'opérateur historique consacré à l'état de la concurrence et publié en mars 2007 (Wettbewerbsbericht, 2007). Cela étant, l'effet de taille joue encore largement en faveur de Deutsche Bahn AG et sa filiale fret Railion Deutschland AG, dont les parts de marché s'établissaient à $83,6 \%$. Mais depuis 2002, ses concurrents ont vu passer leurs parts de 4,8\% à 16,4\%. Dans le secteur du fret, ils seraient au nombre de 97 selon Destatis.

Structure des concurrents de Deutsche Bahn AG oeuvrant dans le fret en RFA (2005)

\begin{tabular}{|l|c|ccc|}
\hline & Total & $\mathbf{1}$ & Nombre de salariés & $\mathbf{5 0}$ \\
Sociétés & 97 & 35 & $\mathbf{1 0}$ à $\mathbf{4 9}$ & 21 \\
Flotte & 105947 & 201 & 41 & 104415 \\
Locomotives & 3169 & 62 & 1331 & 2906 \\
Wagons & 102778 & 139 & 201 & 101509 \\
Effectif & 27972 & 143 & 1130 & 26924 \\
Conducteurs & 11944 & 39 & 905 & 11642 \\
Agents techniques & 12273 & 43 & 263 & 11899 \\
Administratifs & 3755 & 61 & 331 & 3383 \\
\hline
\end{tabular}

Source des données : Destatis, Wirtschaft und Statistik, 8/2007

Mais cette photographie fournie par Destatis, si elle a le mérite d'exister, reste largement incomplète, reflétant les lacunes typiques dans la prise en considération d'un marché en cours de constitution. Le fret ferroviaire n'étant pas non plus considéré comme une branche (ni non plus la logistique, d'ailleurs), les multiples fédérations existantes dans le secteur ne disposent que de données très catégorielles. La multitude des acteurs réglementaires en charge du ferroviaire ne facilite pas, elle non plus, la collecte de données.
Réseau ferré allemand : un maillage conforme au polycentrisme des activités et à l'ouverture au commerce mondial

Part de marché des concurrents de Deutsche Bahn AG : 16,4 \%

La structure du marché du fret est méconnue des statistiques 


\section{Les étapes de la libéralisation du marché ferroviaire dans l'UE et en Allemagne}

\section{- Le contexte européen}

La directive 91/440 CE du 29-07-1991 « relative au développement des chemins de fer communautaires » pose les jalons de la libéralisation du secteur. Son objectif étant de doter l'économie européenne d'infrastructures et services permettant la mobilité requise par l'intégration des activités, elle vise l'accessibilité de tous les réseaux nationaux aux opérateurs d'un autre Etat membre (interopérabilité, non discrimination) et prône à cet effet, pour les sociétés de chemins de fer encore publiques des Etats membres, la séparation de la gestion du réseau d'infrastructures et de son exploitation commerciale. Cette séparation vise notamment le désendettement de ces sociétés pour agir à deux niveaux : d'une part permettre aux opérateurs d'investir dans l'amélioration de la qualité de leurs services techniques et commerciaux, d'autre part, permettre aux gestionnaires publics des réseaux (Etat, société $a d$ hoc) d'effectuer les investissements nécessaires à l'entretien, la modernisation et l'extension des infrastructures.

Malgré l'adoption d'une série de textes complétant ou précisant cette directive, il n'existe jusqu'à aujourd'hui aucun cadre réglementaire organisant de manière globale les activités d'exploitation ferroviaires au sein de l'UE. Néanmoins, la directive de 1991, de même que la directive 95/18/CE instaurant un régime de licences pour les sociétés du rail et la directive 95/19/CE concernant la répartition des capacités d'infrastructure et la tarification de l'infrastructure ferroviaire, après avoir subi diverses modifications, ont été rassemblées au printemps 2001 dans le " $1^{\text {er }}$ Paquet ferroviaire ", suivi d'un second entré en vigueur en 2004 et d'un troisième, dont les actes législatifs ont été publiés à la fin 2007 (JO de l'UE, n ${ }^{\circ} 315$ du 03-12-2007). Les principaux points importants sont aujourd'hui réglementés. Ainsi, la non-discrimination de l'accès au réseau dans un autre Etat membre a mené progressivement, depuis 1993, à l'ouverture totale à la concurrence du marché communautaire du fret au 01-01-2007 (l'ouverture du trafic voyageurs est prévue pour 2010). La même garantie s'est traduite par l'instauration d'un régime d'autorisation applicable à toute société désireuse d'opérer dans le ferroviaire ; ces autorisations, accordées par chaque Etat membre sont reconnues par tous les autres. De même, la pratique des péages comme la répartition des capacités sont contrôlées dans chaque Etat membre par une autorité de régulation. Le Conseil Transports qui s'est réuni le 07-04-2008 à Luxembourg réaffirme l'importance vitale pour l'économie de l'UE d'un "réseau ferroviaire européen qui favorise une circulation efficace » du fret intérieur et international (Communiqué publié à l'issue du Conseil).

\section{-Les étapes en Allemagne}

En septembre 1989, la perte de vitesse du rail dans le modal mix, de même que l'endettement croissant lié aux infrastructures ferroviaires (sur fond de dette publique) incitent le gouvernement fédéral à envisager une réforme structurelle pour remédier durablement à ces problèmes. La chute du Mur précipite les décisions, d'autant que l'Unité (scellée en octobre 1990) implique la fusion des deux sociétés publiques Bundesbahn et Deutsche Reichsbahn, toutes deux déficitaires. Le contexte est doublement propice aux réformes : à l'échelon communautaire comme à l'échelon national, dans le fil de la désétatisation des services d'intérêt économique général engagée à l'été 1989 pour les services postaux et les télécommunications.

Une Loi sur la réorganisation des chemins de fer (Eisenbahnneuordnungsgesetz, ENeuOG) est adoptée le 17-12-1993 et entrée en vigueur en 1994, prévoyant quatre axes de réforme : désendettement, désétatisation/privatisation de l'administration publique, libéralisation du marché et décentralisation des transports en commun et liaisons régionales (transfert des compétences aux Länder). Elle fédère cinq lois réglementant ces aspects spécifiques : Loi générale sur les chemins de fer (Allgemeines Eisenbahngesetz, AEG), Loi de fusion et de réorganisation des chemins de fer fédéraux (Gesetz zur Zusammenführung und Neugliederung der Bundeseisenbahnen, BEZNG), Loi portant création d'une société Deutsche Bahn AG (Deutsche Bahn Gründungsgesetz, DBGrG), Loi relative à l'administration fédérale du trafic ferroviaire (Gesetz über die Eisenbahnverkehrsverwaltung des Bundes, BEVVG) et Loi de régionalisation des transports en commun (Regionalisierungsgesetz, RegG). En application de ces dispositions est créée une société d'exploitation de droit privé, indépendante du gouvernement : la Deutsche Bahn AG, dont le Bund détient indirectement et provisoirement la totalité du capital. Le patrimoine et sa gestion, ainsi que la mission de contrôle et d'autorisation des sociétés de chemin de fer sont confiées à deux administrations externalisées, respectivement : Bundeseinsenbahnvermögen (BEV) et Eisenbahnbundesamt (EBA). La dette de DB et DR (34,4 milliards $€$ en 1994) est apurée par la BEV ; lors de sa création, Deutsche Bahn AG est donc désendettée. Au 01-01-1999, une seconde étape restructure cette société. Ses anciennes directions sont transformées en sociétés par actions : DB Reise\& Touristik AG (trafic voyageurs), DB Regio AG (transports en commun et liaisons régionales), DB Netz AG (réseau), DB Stationen\&Service (gares passagers) et DB Cargo AG (fret), rebaptisée depuis Railion Deutschland AG. Elles sont chapeautées par la Deutsche Bahn AG qui a statut de holding.

Les sociétés d'exploitation sont toutes vouées à la privatisation. La majorité du capital de DB Netz AG, en revanche, doit rester aux mains du Bund puisque, dans le cadre de la réforme de 1994, celui-ci a conservé la propriété des activités liées à "la construction [et] l'entretien... des voies ferrées" (art. 87e de la Loi fondamentale). Cette architecture, de même que le statut particulier de DB Netz AG, enveniment les débats sur la procédure concrète à suivre pour la privatisation de Deutsche Bahn AG, ce qui explique qu'aucune décision n'ait été prise depuis l'ouverture des négociations en 2002. En principe, le contrat de coalition du gouvernement Merkel prévoit l'entrée en bourse de l'opérateur durant son mandat, mais la décision achoppait jusqu'ici sur l'opposition de la gauche du SPD. Un compromis s'est néanmoins dessiné à la fin avril 2008: conformément à la recommandation formulée tant par la Commission européenne que par la Commission des monopoles allemande, les activités liées au réseau seraient sorties du périmètre de Deutsche Bahn pour rester publiques. Les autres sociétés, en charge de l'exploitation commerciale (fret et trafic voyageur), devraient voir leur capital partiellement ouvert. Si le parlement vote le projet, 24,9\% du capital de cette Deutsche Bahn reconfigurée seront mis en bourse à la fin 2008.

La gestion du régime d'autorisation des sociétés de chemin de fer a été confiée aux Länder, en application de leur souveraineté en matière de pouvoir administratif sur leur territoire. Le type des autorités compétentes est donc très varié, allant d'un ministère de l'Intérieur (Bade-Wurtemberg, par exemple) à un ministère ayant en charge les transports (Bavière, Brandebourg, Rhénanie du Nord-Westphalie notamment), un ministère de l'Economie (Sarre ou Basse-Saxe) ou encore, au sein du Land de Hesse, à la présidence des districts régionaux. Les sociétés comptant couvrir l'ensemble du territoire allemand s'adressent, elles, à l'EBA à l'échelon fédéral. Quant au contrôle du libre accès aux infrastructures, comme de la conformité de la pratique des péages, il a été confié le 01-01-2006 à la Bundesnetzagentur für Elektrizität, Gas, Telekommunikation, Post und Eisenbahnen (BNetzA), l'ancienne autorité de régulation des postes et télécommunications qui avait vu à cette occasion s'étendre ses domaines de compétences aux secteurs de l'électricité, du gaz, et aux chemins de fer. 
Enfin, l'histoire des chemins de fer a contribué elle aussi à la complexité du secteur; nombre d'opérateurs publics ou privés existaient en effet outre-Rhin dès avant l'ouverture du marché ferroviaire à la concurrence en 1994. Le seul acteur ayant à ce jour tenté de dresser le portrait de la concurrence est Deutsche Bahn AG (Wettbewerbsbericht 2007). Or, remarque la Commission des monopoles, « rien ne permet de juger de sa fiabilité » quant à l'approche d'ensemble. Cela étant, quelques grands opérateurs émergent, dont la société Häfen und Güterverkehr Köln AG qui exploite les infrastructures portuaires de Cologne, la SBB Cargo Deutschland, filiale des chemins de fer suisses, la Rail4Chem, récemment rachetée par Veolia, ou cette société Import Transport Logistik (ITL, Dresde) sur laquelle la SNCF vient de lancer une OPA. Quant à l'autorité de régulation, la $B N e t z A$, elle a certes engagé en 2006 une veille du marché, mais celle-ci est loin d'être achevée. En attendant, il faut donc se contenter d'estimations qui portent le nombre total de sociétés du rail à 1000 environ.

La plupart d'entre elles sont publiques. Au XIX $X^{e}$ siècle, le chemin de fer était né à l'échelon régional, et ces activités ont été nationalisées au cours des années 1920. Elles relèvent aujourd'hui constitutionnellement des prérogatives des Länder. II existe ainsi, du fait de l'histoire, un certain nombre de sociétés de chemins de fer appartenant aux Länder, comme celles desservant les ports d'Aschaffenburg et de Ratisbonne (Land de Bavière) ou la société Altona-Kaltenkirchen-Neumünster (Hambourg et Schleswig-Holstein). La plupart des communes possèdent elles aussi leurs sociétés de chemin de fer, qu'elles exploitent conformément à leur mission de service public qui inclut l'approvisionnement en eau, énergie, la gestion des déchets et des transports en commun. Le plus souvent, à l'origine, ces sociétés communales de chemin de fer avaient été créées expressément pour l'industrie locale. Certaines d'entre elles ont étendu leurs activités de fret à l'échelon suprarégional, dont celles de Hambourg (desserte du port) Cologne (desserte des plate-formes fluviales et maritimes ; wagons citernes et conteneurs) ou de Hanovre (conteneurs).

Par ailleurs, le Bund est compétent pour les chemins de fer fédéraux, desservant l'ensemble du territoire, et pour l'attribution de licences aux sociétés étrangères. Or la Deutsche Bahn possède une douzaine de filiales dans les Länder ou les communes, dont elle est actionnaire majoritaire, ce qui confère à ces sociétés un statut fédéral. Dans le segment du fret, il s'agit de la Mitteldeutsche Eisenbahn Gesellschaft (liaisons dans la région de Halle et Leipzig ; transports nationaux de ciment) et de la RAG Bahn und Hafen (prestataire pour le groupe Ruhrkohle AG et propriétaire d'un réseau ferré de $430 \mathrm{~km}$ dans la Ruhr; Deutsche Bahn AG a récemment pris une part majoritaire dans son capital). II faut ajouter à cet ensemble les sociétés de chemins de fer intégrées aux groupes industriels comme BASF, Bayer ou Thyssen Krupp (liaisons inter-sites), ainsi que celles appartenant à des sociétés d'exploitation portuaires, par exemple la Duisport rail GmbH qui dessert le port fluvial de Duisburg.

150 sociétés privées œuvrent dans le fret ferroviaire
Sur le total des sociétés agissant dans le fret depuis l'intensification de la concurrence à la fin 1997,
seules 150 sont privées. Toutes n'ont pas leurs propres infrastructures. La Commission des mono-
poles les classe en 5 catégories :
- sociétés n'appartenant pas au Bund et assurant des liaisons nationales ou transfrontières, pour
partie en coopération avec des sociétés 'tierces' (étrangères) ;
- sociétés nouvellement créées par des transporteurs (souvent en coopération avec des presta-
taires logistiques);
- sociétés fondées par des particuliers (souvent par management buy out) ;
- sociétés étrangères ayant racheté une entreprise allemande (exemple : l'ex Deutsche Eisenbahn-
gesellschaft mbH appartient aujourd'hui à Veolia Verkehr GmbH);
- sociétés conjointes créées par des opérateurs tiers et allemands.

Une autre difficulté pour aborder le marché du fret ferroviaire réside dans le fait que « le transport de marchandises est partie constitutive de la production », ainsi que le formule la Commission des monopoles. Quel que soit le mode, le transport
Une structure très polycentrique et une majorité de sociétés publiques

Le choix du mode obéit à une demande complexe 
Fluvial/ferroviaire : concurrents pour le seul fret pondéreux

Concurrence rail/route seulement au-delà de $50 \mathrm{~km}$

Rail pour le fret pondéreux route pour le transport groupé n'est en effet « qu'un moyen mis en œuvre pour réaliser un objectif.... Ce n'est donc pas le transport en tant que tel qui crée valeur et profit, mais la possibilité de transporter un bien en un autre lieu pour l'y employer dans la production ou l'y consommer ». II en découle qu'il faut considérer le marché du fret sous l'angle de la demande - d'une demande indirecte. De ce fait, l'acteur principal du marché est le transporteur, car c'est lui qui a la maîtrise des divers modes. Le choix du mode le mieux adapté aux besoins du commanditaire est ensuite effectué par le chargeur ou le commissionnaire, ce dernier pouvant par ailleurs exercer aussi le rôle de transporteur. La détermination du mode obéit à deux critères : à la relation coûts-avantages en fonction du bien à transporter et aux caractéristiques ou qualités systémiques inhérentes à chaque mode. C'est sur la base de ces critères que les divers modes sont mis en concurrence.

Il faut donc considérer le 'marché' ferroviaire sous deux angles : celui de la concurrence intra-modale (au sein du même mode) et, surtout, dans le contexte plus global de la concurrence intermodale (entre les divers modes). Et examiner, comme le fait la Commission des monopoles, la relation coûts-avantages selon la nature des biens considérés. En ce qui concerne le couple fluvial/ ferroviaire, ces deux modes n'entrent en concurrence que pour les marchandises pondéreuses 'de base' (fret d'un poids supérieur à 100 t) qui se caractérisent par une faible valeur à la tonne, comme les céréales, le bois, le charbon, les minerais, les matériaux de construction, les produits pétroliers, chimiques, les engrais ou déchets recyclables. Les capacités d'un train complet (1 $000 \mathrm{t}$ en moyenne) et d'une péniche sont comparables. De même, les deux modes offrent à ces marchandises la même dégressivité des coûts fixes, mais avec un désavantage pour le rail dont les charges administratives et salariales sont plus élevées que dans le fluvial. La relation s'inverse en faveur du ferroviaire si on prend en compte un autre critère : le réseau et ses possibilités d'interconnexion. Cela étant, des industries comme la sidérurgie se sont depuis toujours localisées à proximité des fleuves et canaux, ce qui contribue à expliquer la position de leader d'un port comme Duisburg, point nodal intermodal par excellence. Le transfert du rail au fluvial est limité en outre par l'étiage des fleuves, ainsi que l'ont révélé les basses eaux du Rhin durant la canicule de 2003 ; non seulement le rail a bénéficié alors d'un effet de substitution, mais il a su fidéliser depuis une large partie de ses clients. La concurrence entre ces deux modes n'est donc réellement effective qu'aux abords des voies de navigation intérieures, principalement dans le bassin du Rhin et de son réseau de canaux et affluents.

La concurrence entre le rail et la route se présente sous un angle plus complexe. Si on inclut dans le transport routier les liaisons inter-sites de l'industrie, il appert que près de la moitié du total du fret en volume (le quart en tkm) est acheminé sur des distances inférieures à $50 \mathrm{~km}$; les 'stocks' des entreprises industrielles allemandes se révèlent très nomades... Dans ce segment, il ne peut donc y avoir de concurrence intermodale.

Tout à l'inverse des distances supérieures. Mais il faut distinguer ici trois segments. Dans celui des biens 'de base', seul le rail offre les capacités requises (train complet), et il n'y a pas de substitution possible par la route - sauf peut-être si sont mis en service les "giga-liners", ces poids lourds de $25 \mathrm{~m}$ et d'une charge utile allant jusqu'à 60 tonnes. Mais l'évolution de la structure des activités, dans laquelle le transport de biens intermédiaires gagne en importance, ne plaide guère en faveur d'une forte rentabilité de la route pour des biens comme le bois ou les minerais. A l'inverse, dans le segment du transport groupé (du colis à la palette), qui ne peut s'effectuer qu'en complémentarité avec la route pour le préet post-acheminement, le rail n'est guère compétitif, car pas assez souple (il ne répond notamment pas à la demande d'un service 24 heures sur 24). La Bundesbahn, qui s'était diversifiée dans ce segment, avait décidé, après sa transformation en Deutsche Bahn AG, d'externaliser ces activités ; mais elle s'est désengagée en 1999 du joint venture créé en 1995 avec le groupe Thyssen Haniel Logistic et cédé depuis à $A B X$ (filiale des chemins de fer belges). 
Une substitution n'est possible que dans le troisième segment, celui des chargements mixtes conteneurs/caisses mobiles, bien que le rail requière une logistique plus complexe pour les chargements/déchargements (équipements de triage notamment) qui réduit la flexibilité de ce mode et se traduit par un coût de manutention et une durée d'acheminement plus élevée. Le choix du ferroviaire implique également que l'expéditeur et le destinataire disposent d'un réseau de voies ferrées. En revanche, le transport combiné, puisqu'il inclut pré- ou postacheminement routier, force la complémentarité des deux modes, sachant toutefois qu'il renchérit le transbordement et, surtout, qu'il se heurte à une série d'obstacles comme des horaires de travail peu flexibles dans les plate-formes ou les difficultés rencontrées pour emprunter le réseau ferré d'un Etat voisin. La Commission des monopoles estime de ce fait que le combiné n'est compétitif sur le rail qu'au-delà d'une distance de $350 \mathrm{~km}$, donc à l'international surtout; et, à l'échelon national, principalement pour desservir l'hinterland portuaire.

Mais pour l'heure, la compétitivité du rail est freinée par «les méthodes de production des sociétés de chemin de fer " (Commission des monopoles, 2007), encore largement imperméables à l'approche flexible du supply chain management que requièrent des process industriels orientés aujourd'hui non seulement just in time, mais de plus en plus souvent just in sequence. Facteur aggravant, le nombre de voies de raccordement reliant les réseaux privés des industriels au réseau ferré fédéral est tombé, entre 1990 et 2002, de 16000 à 5500 . De surcroît, Deutsche Bahn AG s'était largement retirée du transport en wagon isolé dans le cadre de son programme de rationalisation interne MORA C. Cette situation a incité un certain nombre de groupes industriels à renouer avec le rail, comme ce consortium Kombiterminal Ludwigshafen $\mathrm{GmbH}$, créé autour du chimiste BASF, et qui a construit sa propre plate-forme en 2000, d'une capacité de 300000 UTI à ce jour.

La distinction de ces segments du transport révèle que la concurrence intramodale (entre acteurs œuvrant dans le rail) est tributaire de deux tendances : l'évolution de la structure du fret et l'effet logistique lié aux process. Elle ne peut donc concerner d'une part que certains types de marchandises: les classiques biens 'de base' transportables par trains complets ou par wagons groupés. Les nouveaux entrants négligeront donc dans cette catégorie les biens dont le volume diminue du fait des mutations de l'économie (par exemple le charbon) et privilégieront à l'inverse ceux qui offrent une relation coûts-avantages favorable et un net potentiel de développement, comme les engrais, les matériaux de construction, et surtout les hydrocarbures ou les produits chimiques (rappelons que la chimie est un des secteurs clefs de l'économie allemande).

D'autre part, sous l'effet-logistique, la concurrence intra-modale gagne du terrain dans la catégorie des biens transportables par UTI, comme l'équipement automobile (plus généralement tous les biens intermédiaires et d'investissement), mais seulement sur les longues distances, seules à même de compenser les coûts élevés de manutention. L'expansion de ce segment est toutefois limitée à l'heure actuelle par la faible interopérabilité du trafic transfrontières à laquelle tente de remédier la Commission européenne dans le cadre de sa complexe politique d'infrastructures de transports et de mobilité. Dans ces deux segments des prestations logistiques, les concurrents de Deutsche Bahn AG sont donc soit les grands transporteurs, soit les industriels et/ou leurs sous-traitants logistiques. Ils se concentrent dans ces Länder qui sont les principaux sites industriels d'Allemagne : Rhénanie du Nord-Westphalie (la plate-forme logistique par excellence), BaseSaxe, Bade-Wurtemberg et Bavière.

A ces entreprises ferroviaires prestataires, il convient d'ajouter les sociétés gestionnaires d'infrastructures. La BNetzA estime le nombre des premières à 330 , celui des secondes à 760 , mais sans distinguer entre trafic voyageurs et fret, ni entre statut public et privé. Le seul chiffre fiable est la part de marché globale de l'ensemble de ces sociétés dans le fret ferroviaire : les quelque $16 \%$ avancés par Deutsche Bahn AG. Or les infrastructures lourdes comme le rail relèvent de ce
Concurrence rail/route dans le transport combiné

Mais le rail n'est guère adapté encore au combiné

Concurrence intra-modale surtout dans le trafic conteneurs

La concurrence reste limitée par l'accès au réseau, ... 
... plus au niveau de la gestion des sillons que de la tarification qu'il est convenu d'appeler un «monopole naturel ». Le nombre de concurrents de Deutsche Bahn AG sur ce marché est donc par nature limité, et la concurrence intra-modale ne concerne qu'à la marge la gestion des infrastructures. Le marché pertinent à considérer est dès lors celui des prestations. Et dans celui-ci, la question de la concurrence se concentre moins sur les coûts en amont (électricité ; Deutsche Bahn a ici un avantage compétitif) ou l'accès à la flotte (le segment des loueurs de locomotives ou wagons s'est largement constitué, avec des sociétés comme Dispolock ou Angeltrans) que sur la problématique de l'accès au réseau. Celle-ci se pose à deux niveaux : la gestion des sillons (créneau horaire d'un passage de convoi sur un tracé donné) et leur péage.

De l'avis de la Commission des monopoles, la tarification de l'accès aux sillons (péage) n'est actuellement pas discriminatoire. En effet, la structure spécifique du secteur ferroviaire (la position de quasi-monopole de Deutsche Bahn AG en tant que gestionnaire d'infrastructure et exploitant commercial) et l'existence d'une concurrence intermodale sur le marché du fret (donc la possibilité pour un chargeur de préférer par exemple la route au rail) empêchent la constitution de rentes de monopole. C'est là ce qui distingue foncièrement le rail des autres industries de réseaux. Pour l'opérateur historique, fortement endetté par ses activités réseau, une baisse significative des tarifs de péage fixés par le Décret réglementant l'usage des infrastructures ferroviaires (Eisebahninfrastruktur-Benutzungsverordnung, EIBV) du 03-06-2005 ne présente aucun intérêt, puisqu'en vendant plus de sillons, il perdrait des parts de marché intra-modales, c'est-à-dire au bénéfice de ses concurrents sur le marché des prestations de services ferroviaires. A l'inverse, une hausse permettrait certes de combler son déficit (porté par la filiale DB Netz AG) mais se traduirait invariablement par une chute des parts de marché intermodales du rail, c'est-à-dire au profit essentiellement de la route. Ce fait souligne, soit dit en passant, la contradiction foncière existant entre d'une part l'objectif économique de l'ouverture du ferroviaire au marché (bridée par une régulation non adaptée et, plus encore, par la non séparation entre les activités liées aux infrastructures et à l'exploitation commerciale de celles-ci) et, d'autre part, l'objectif politique de développement du mode rail par rapport à la route (limité pour les mêmes raisons).

La gestion des sillons, en revanche, qui relève des seules pratiques commerciales, permet d'élever des barrières d'entrée aux concurrents. Dans la mesure où c'est l'opérateur historique de réseau qui procède à la planification annuelle des flux (dans le contexte européen), il privilégie en toute logique le service régulier par rapport aux commandes additionnelles, mais il bénéficie surtout d'un temps d'avance favorable à la programmation de ses propres sillons. C'est donc aussi dans la complexité même de la planification des flux que réside une des principales entraves à la concurrence. Cette situation n'ayant pas donné lieu officiellement à conflit jusqu'ici, il semblerait que les nouveaux entrants s'en accommodent, sachant par ailleurs faire jouer la concurrence intermodale, donc en préférant la route au rail...

“ LIBERALISER UN SECTEUR N'EST PAS UNE FIN EN SOI », rappelle la Commission des monopoles, " mais plutôt un moyen pour réaliser une multitude d'objectifs économiques et politiques ». Or peu d'entre eux ont été atteints depuis 1994. La dette liée au réseau public des chemins de fer n'est pas apurée, ce qui réduit la marge de manœuvre du gouvernement fédéral pour investir dans la modernisation des infrastructures. Certes, entre 1994 et 2005, les dépenses du gouvernement fédéral pour l'ensemble du système ferroviaire se sont élevées à 232 milliards $€$, soit un peu plus de 19 milliards $€$ par an. En 2005, elles atteignaient 18,8 milliards $€$, soit $7 \%$ du total des dépenses du Bund la même année. Or la majeure partie d'entre elles était affectée à la dette accumulée par l'ex-Reichsbahn, la société des chemins de fer de l'ex-RDA, fusionnée avec la Bundesbahn lors de l'Unité allemande. Depuis 1994, la Deutsche Bahn AG perçoit quelque 3,7 milliards $€$ par an en moyenne (prêts et subventions) au titre des investissements dans le réseau d'infrastructures (Monopolkommission, 2007). Le plan budgétaire 2007- 
2011 du Bund prévoit 9,5 milliards $€$ de dépenses en moyenne par an pour le ferroviaire (total tous modes confondus : un peu plus de 16,6 milliards $€$ par an, dont quelque 5,5 milliards $€$ pour la route), dont un peu plus de 3,5 milliards $€$ par an seulement pour l'investissement dans les infrastructures, le reste étant affecté à leur entretien (c'est environ le double en moyenne des investissements français sur les mêmes périodes ; Sénat, 2008). Ces dépenses conséquentes sont pourtant insuffisantes notamment pour doubler systématiquement les voies afin de fluidifier le fret. Plus important encore, elles sont largement absorbées par les charges liées à l'histoire et à l'entretien du réseau. Rien ne permet toutefois d'affirmer si l'objectif premier de la réforme ferroviaire : réduire le déficit des budgets publics, a pu être atteint ou non, explique la Commission des monopoles. Quant à l'effet de la privatisation partielle à venir de Deutsche Bahn AG sur la dette, il est tout aussi peu probant, puisque constitutionnellement la charge du réseau relève du Bund. Cette problématique est commune à tous les Etats membres, et le développement de partenariats public-privé est envisagé à l'écheIon communautaire. Mais sous quelle forme et avec quelle efficience?

Second objectif : l'expansion du rail. Il est loin d'être atteint, malgré le dynamisme incontestable du fret ferroviaire outre-Rhin. Malgré l'ouverture précoce du marché allemand en comparaison européenne, le mode rail a longtemps stagné et n'a pu que reconquérir, et en 2006 seulement, la part qu'il avait dans la répartition intermodale avant 1994 (plus de 16\%) - faute d'investissements, mais aussi à cause d'une libéralisation inachevée. Certes, le cadre pour le libre jeu de la concurrence a été mis en place, sous la pression communautaire comme du fait des impératifs nationaux. Mais les décideurs politiques, aux prises plus avec les difficultés à concilier la pluralité des intérêts des acteurs publics en charge du ferroviaire qu'avec des conflits idéologiques sur le service public ou universel du rail, ont jusqu'ici reculé devant les choix politiques stratégiques nécessaires à la libéralisation. Ils ont ainsi opté pour une sorte de compromis qu'on pourrait caractériser ainsi : pourvu qu'il y ait concurrence au niveau de l'exploitation, le déséquilibre public/privé au niveau des opérateurs d'infrastructures importe peu pour l'instant... Or l'évolution du marché du fret et de la logistique dans l'espace européen et allemand rend urgente une décision sur le statut de l'opérateur historique.

La société Deutsche Bahn AG est certes désétatisée, mais toujours publique. Ce fait place l'Etat face à un conflit d'intérêts : actionnaire, il ne peut que désirer la prospérité de sa société, ce qui l'incite, dans sa fonction de régulateur, à maintenir des barrières élevées à l'entrée des concurrents sur le marché afin que Deutsche Bahn AG reste l'acteur majeur du mode ferroviaire. Acteur politique enfin, il doit veiller à créer les conditions physiques et réglementaires propices à la mobilité sur son territoire et, plus généralement, au bon fonctionnement de l'économie ; il doit donc créer les conditions pour que la concurrence intra-modale dynamise le rail et que ce mode, ainsi optimisé, gagne en importance dans un contexte logistique plus global mettant en concurrence les coûts-avantages respectifs des divers modes de transport de marchandises afin d'optimiser la complémentarité de l'ensemble du réseau d'infrastructures allemand (et européen). L'ouverture du marché des transports à la concurrence en est une des conditions, un enjeu vital de surcroît pour la compétitivité future d'une économie aussi tributaire des échanges que l'Allemagne. La création d'un marché communautaire des chemins de fer est d'autant plus vitale pour elle que seules les longues distances et le transport de conteneurs offrent une réelle perspective au mode ferroviaire.

Une politique de modernisation des infrastructures ou le développement d'une stratégie marketing pour le site logistique qu'est Allemagne, ainsi que se propose de le faire le gouvernement fédéral dans le "Masterplan » élaboré conjointement avec les acteurs du secteur en complémentarité avec le "Plan d'action pour la logistique » de la Commission européenne, ne suffisent pas. Pour que la concurrence puisse pleinement déployer ses effets à l'inter- comme à l'intra-modal, le marché des transports doit être libéralisé dans sa totalité. La raison économique plaide ainsi pour une privatisation rapide de Deutsche Bahn AG afin que le mar- 
ché du fret ferroviaire puisse se structurer autour d'une pluralité de prestataires et que le mode rail gagne en compétitivité. Mais comment procéder? S'agissant d'un monopole naturel, le réseau d'infrastructures, intégré à l'opérateur via sa filiale DB Netz AG, n'est pas privatisable, ce qui rend impossible l'introduction en bourse de la holding. DB Netz AG entravant la compétitivité de Deutsche Bahn $A G$, il conviendrait donc de sortir la filiale de son périmètre, à l'exemple du modèle français, ce qui transformerait la holding en simple prestataire de services. Or la décision politique avait longtemps achoppé sur un point annexe : le double positionnement de l'opérateur sur le fret et le transport de voyageurs. Ainsi, c'est aussi le délicat débat politique sur les services d'intérêt général en Allemagne comme dans l'UE qui bride le potentiel du fret ferroviaire, dont la nature de marché ne prête, elle, pas à débat. Néanmoins, à la fin avril 2008, les deux formations de la coalition fédérales sont parvenues à un accord: les infrastructures resteront dans le domaine public, les activités d'exploitation (fret et voyageur) seront partiellement privatisées. Reste à reconfigurer Deutsche Bahn AG.

En Allemagne, à l'inverse de la France, le système ferroviaire du transport de marchandises n'est pas sous-dimensionné, loin de là, et il est potentiellement concurrentiel. Mais, pour diverses raisons, l'Allemagne peine à l'optimiser. Le choix timoré d'une structuration des acteurs ni publique ni réellement privée ne résiste pas aux impératifs de compétitivié pour la première plate-forme logistique de l'UE. "L'absence d'harmonisation au niveau européen ne doit pas servir de prétexte à interrompre le processus de libéralisation », insiste la Commission des monopoles. En effet, " ce n'est que si la concurrence intra-modale fonctionne que le rail pourra gagner en compétitivité et optimiser la place qui lui revient dans la concurrence inter-modale ».

\section{Indications bibliographiques}

ALLIANZ PRo SCHIENE, Mehr Bahn wagen. 13 erfolgreiche Verlagerungsbeispiele aus dem Güterverkehr, mars 2007 (www.allianz-pro-schiene.de)

BOURGEOIS I. (dir), Allemagne : compétitivité et dynamiques territoriales, Travaux et Documents du CIRAC, 2007

BUNDESAMT FÜR GÜTERVERKEHR (BAG), Marktbeobachtung Güterverkehr, Bericht Herbst 2007, novembre 2007

BUNDESMINISTERIUM FÜR VERKEHR, BAU UND STADTENTWICKLUNG (BMVBS), Masterplan Güterverkehr und Logistik - Entwurf, mars 2008 (www.bmvbs.de)

BUNDESMINISTERIUM FÜR VERKEHR, BAU UND WOHNUNGSWESEN (BMVBW), Bundesverkehrswegeplan 2003 (www.bmvbs.de)

BUNDESNETZAGENTUR, Jahresbericht 2006, février 2007

BVU, DLR, ISL, Gleitende Mittelfristprognose für den Güter- und Personenverkehr. Mittelfristprognose Winter 2006/2007, janvier 2007

COMMISSION EUROPEENNE, RTE-T, axes et projets prioritaires 2005, 2005, et La politique européenne des transports à l'horizon 2010 : l'heure des choix, Livre blanc, 2001

Conclusions du Conseil sur la Communication de la Commission intitulée "Vers un réseau ferroviaire à priorité fret ", $281^{\mathrm{e}}$ session du Conseil Transports, Télécommunications et Energie, Luxembourg, 07-04-2008 (communiqué de presse ; www.europa.eu) DESTATIS, Wirtschaft und Statistik, $n^{\circ} 6 / 2007$ et $n^{\circ} 8 / 2007$

DeUTSCHE BAHN AG, Wettbewerbsbericht 2007, mars 2007 (www.db.de)

DIHK, Verkehrspolitik der Zukunft-Wachstum sichern durch Mobilität. Resolution des DIHK-Vorstandes, juin 2007

EUROSTAT, «Unitarisation du transport de marchandises en Europe, 2005 », Statistiques en bref, $n^{\circ} 20 / 2008$

IFMO (ed), Verkehrsinfrastruktur-Benchmarking Europa, septembre 2007 (www.ifmo.de) ITP/BVU, Prognose der deutschlandweiten Verkehrsverflechtungen 2025, novembre 2007 KLAus P., KILLE C., Die Top 100 der Logistik 2006, Fraunhofer IIS/ATL, février 2007 MONOPOLKOMMISSION, Wettbewerbs- und Regulierungsversuche im Eisenbahnverkehr. Sondergutachten, $\mathrm{n}^{\circ}$ 48, avril 2007, et Die Privatisierung der Deutschen Bahn AG, Sondergutachten, $\mathrm{n}^{\circ} 46$, septembre 2006 ( www.monopolkommission.de)

Progtrans, Abschätzung der langfristigen Entwicklung des Güterverkehrs in Deutschland bis 2050, Schlussbericht, mai 2007 (www.progtrans.com)

Reiner D., Billout M., BIWER C., Rapport d'information fait au nom de la commission des Affaires économiques par la mission d'information portant sur le fonctionnement et le financement des infrastructures de transport terrestres, n¹96, Sénat, février 2008 www.ferrmed.com 